Reprod. Nutr. Dévelop., 1983, 23 (5), 927-941.

\title{
Etude histologique et ultrastructurale de l'organogenèse et de la différenciation post-embryonnaires de l'appareil génital d'Helix aspersa Müller
}

\section{J. ENÉE, Bernadette GRIFFOND}

\author{
Laboratoire de Zoologie et Embryologie, L. A. CNRS $n^{\circ} 040310$ \\ Faculté des Sciences et des Techniques \\ Place Maréchal-Leclerc, 25030 Besançon Cedex, France.
}

Summary. An histological and ultrastructural study of the organogenesis and postembryonic differentiation of the genital apparatus of Helix aspersa Müller.

The post-embryonic genital apparatus of the snail Helix aspersa has been investigated using light and electron microscopical techniques. At hatching, the gonad contained both stem and germinal cells. During the first month, male and female cells differentiated while the ultrastructural analysis of different parts of the tract still showed an undifferentiated epithelial cell type.

\section{Introduction.}

Sujet de controverses durant des décennies, le développement de l'appareil reproducteur des Mollusques Gastéropodes Pulmonés a fait l'objet de nombreux travaux analysés par Martoja (1964) et Tardy (1970). Plusieurs problèmes demeurent lorigine des différentes parties du complexe génital, modalités de différenciation, ...) et cependant, mis à part les travaux de Hochpöchler (1979), Hochpöchler et Kothbauer (1979), peu d'études récentes sont consacrées aux aspects précoces des voies génitales. Dans le cadre de recherches sur la différenciation sexuelle de l'Escargot Helix aspersa (Enée et al., 1977 ; Gomot et Enée, 1980), nous nous sommes intéressés à l'aspect ultrastructural de la gonade à l'éclosion (Griffond et Bride, 1981) et de certaines glandes annexes du tractus génital (Courtot et Gomot, 1982). La présente note est une description histologique et ultrastructurale de la gonade et du tractus génital d'Helix aspersa au cours du premier mois de vie post-embryonnaire.

\section{Matériel et méthodes.}

I. Animaux. - Après avoir été prélevés dans la nature, les Escargots adultes nécessaires à l'obtention de pontes sont placés en terrarium, soumis à des 
conditions naturelles d'éclairement et nourris de laitues et de carottes avec un ajout hebdomadaire de carbonate de calcium « blanc de Troyes " (Crowell, 1973). Les pontes sont recueillies dans des bocaux renfermant de la terre préalablement stérilisée et maintenue constamment humide ; elles sont conservées à l'obscurité, à une température de $20^{\circ} \mathrm{C}$. L'éclosion survient environ 10 jours après la ponte.

Les animaux étudiés proviennent de l'éclosion simultanée en été de 5 pontes différentes. Durant le premier mois de la vie post-embryonnaire, tous les deux jours, 5 Escargots, débarrassés de leur coquille, sont fixés puis inclus à la paraffine ou dans des résines.

II. Techniques. - Pour l'étude histologique, les animaux sont fixés au liquide de Bouin-Hollande sublimé, inclus à la paraffine et colorés au trichrome hématoxyline de Groat, fuchsine acide-ponceau et bleu d'aniline (Martoja et Martoja, 1967).

Pour l'étude ultrastructurale, les Escargots entiers ou l'appareil génital sont fixés pendant $1 \mathrm{~h}$ dans une solution de glutaraldéhyde à $2 \%$, tamponnée au cacodylate de sodium $0,1 \mathrm{M}$ (la pression osmotique est ajustée avec $\mathrm{NaCl}$ à $250 \mathrm{mOsM})$. Après rinçage dans un mélange à volumes égaux de cacodylate de sodium $0,1 \mathrm{M}$ et $\mathrm{NaCl} 0,16 \mathrm{M}$, ils subissent une post-fixation $\mathrm{d}^{\prime} 1 \mathrm{~h}$ dans le tétroxyde d'osmium à $2 \%$ tamponné au cacodylate de sodium (PO= 250 mOsM). Après déshydratation, selon la méthode de Luft (1961), les inclusions sont réalisées dans le mélange épon-araldite utilisé par Anderson et Ellis (1965) en ramenant la proportion de DMP 30 à $1,7 \%$ suivant la méthode de Mollenhauer (1964). Les coupes semi-fines de 0,5 à 1,5 $\mu \mathrm{m}$ d'épaisseur sont colorées au bleu de toluidine (Trump et al., 1961), les coupes ultra-fines contrastées à l'acétate d'uranyle à 3-4 \% dans le méthanol puis au citrate de plomb selon Reynolds (1963). La mise en évidence du glycogène est réalisée par la technique à l'acide periodique-thiocarbohydrazide-protéinate d'argent d'après Seligman, modifiée par Thiéry et Rambourg (1974).

\section{Résultats.}

\section{Aspect macroscopique de l'appareil génital.}

Durant le premier mois de vie post-embryonnaire, l'appareil génital est très rudimentaire. A un mois (fig. 1, PI. I), il est constitué par un cordon filiforme blanchâtre, terminé à une extrémité par un renflement étoilé, digité, qui représente la gonade et à l'autre extrémité par le bourgeon pénien muni de son muscle rétracteur. A un tiers environ de l'ébauche du tractus, du côté de l'ovotestis, une petite protubérance indique la future glande à albumen; elle sépare la zone postérieure très mince ou canal de l'ovotestis (futur canal hermaphrodite) d'une région moyenne (fig. 2 et 3 , PI. I) qui correspond au futur spermoviducte et aux canaux accessoires (branche copulatrice et canal de la bourse copulatrice). Ces deux canaux, absents à la naissance, se forment au cours du premier mois de la vie. 


\section{Description des différentes zones de l'appareil génital.}

1) Ebauche du pénis. - A l'éclosion et au cours des premiers jours qui suivent la naissance, l'ébauche pénienne se présente sous forme d'une évagination épithéliale du conduit génital primaire. Au sein de l'épithélium unistratifié, on relève de nombreuses divisions cellulaires qui concourent à l'établissement d'un épithélium pseudo-stratifié bien visible dans le bourgeon pénien ovoïde des animaux âgés d'un mois. En ultrastructure (fig. 4, PI I), le noyau allongé des cellules de cette ébauche est caractérisé par une disposition en travées des masses d'hétérochromatine. Le cytoplasme, abondamment chargé de particules de glycogène, renferme de nombreuses mitochondries aux deux pôles cellulaires, plus particulièrement dans la région apicale. A cette extrémité de la cellule, la membrane est garnie de microvillosités très courtes bordant une lumière en forme de fente. Des jonctions du type "adherens " suivies de jonctions septées sont observées dans la zone supérieure des membranes latérales. Une lame basale circonscrit l'épithélium et le sépare du mésenchyme constituant la gaine du pénis.

2) Ebauche de la glande à albumen. - En microscopie photonique, à 16 jours une dilatation du conduit génital et un épaississement de l'épiíhélium annoncent l'emplacement de la future glande à albumen. Chez un animal âgé de 23 jours (Fig. 3, PI. I), celle-ci se présente sous forme d'un petit massif cellulaire qui résulte d'une prolifération latérale de cellules épithéliales de l'ébauche tubulaire du spermoviducte. Cet aspect précoce de la glande précède le stade tubulaire simple de Courtot et Gomot (1982) qui décrivent en ultrastructure la cellule épithéliale indifférenciée avant la formation des premiers grains de sécrétion.

3) Ebauche de la gonade. - La gonade et le canal hermaphrodite, à la naissance et au cours de la première semaine de vie post-embryonnaire ayant été décrits par Griffond et Bride (1981), nous n'insistons pas sur les aspects histologiques et ultrastructuraux des différentes catégories cellulaires dont nous suivons l'évolution pendant le premier mois.

a) Morphologie. A l'éclosion, la gonade est un cordon plein $(80$ à $100 \mu \mathrm{m} \times$ 15 à $20 \mu \mathrm{m}$ ) situé à l'extrémité du tortillon, entre la poche de résorption de l'albumen et la paroi du corps, en communication avec le canal hermaphrodite creusé d'une lumière relativement large à ce niveau. Vers le septième jour, ce cordon commence à se ramifier et après deux semaines, la gonade est un petit massif translucide, étoilé, qui insinue entre les tubules de l'hépatopancréas de longs prolongements, eux-mêmes plus ou moins ramifiés. En même temps, à partir de la zone de jonction avec le canal hermaphrodite, une lumière se met progressivement en place, par écartement des cellules.

b) Différenciation. La gonade d'animaux à l'éclosion renferme toujours deux types cellulaires bien distincts: des cellules à aspect somatique ou cellulessouches, à noyau irrégulier riche en hétérochromatine et cytoplasme 

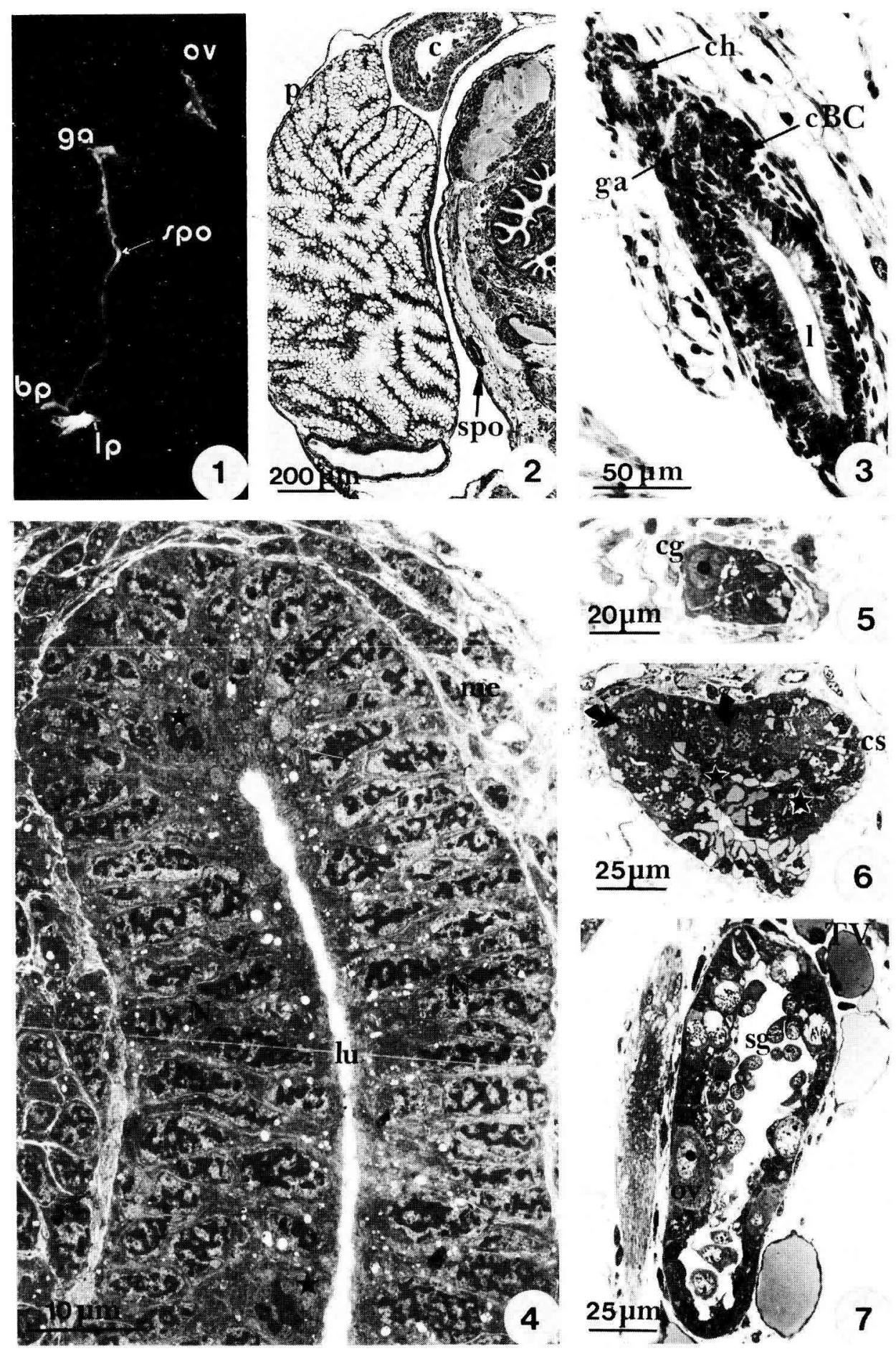
abondamment pourvu de réserves polysaccharidiques et des cellules germinales claires. Deux cas peuvent se présenter :

- ou bien la gonade a conservé un aspect embryonnaire (Gomot et al., 1982) avec des cellules germinales volumineuses et indifférenciées (fig. 5, PI. 1).

- ou bien son évolution est plus avancée et dès la première semaine, des ovocytes, allongés parallèlement à la paroi, sont reconnaissables (Griffond et Bride, 1981). D'autres cellules germinales, orientées de façon variable, sont vraisemblablement à l'origine des cellules de la lignée mâle, identifiables seulement à partir de la deuxième semaine (fig. 6, PI. I).

Après 18 jours, tous les types cellulaires de l'ovotestis sont présents (ovocytes, cellules folliculeuses, cellules nourricières et spermatogonies). Vers la fin du premier mois de vie post-embryonnaire, quelques cellules mâles ont atteint le stade spermatocyte I tandis que de nombreux ovocytes sont en prévitellogenèse (fig. $7, \mathrm{PI}$. I).

\section{4) Ebauche du spermoviducte.}

a) Etude histologique. L'ébauche du spermoviducte est décelable en microscopie photonique dès la naissance. Chez des animaux âgés d'un jour (fig. 1, PI. II), c'est un tube aplati bordé (sur coupe transversale) de 10 à 15 cellules épithéliales, que l'on repère assez facilement dans le plancher de la cavité

\section{PLANCHE I.}

FIG. 1. - Aspect macroscopique de l'appareil génital d'un Escargot âgé d'un mois. Diamètre de la coquille : $8,7 \mathrm{~mm}$ (bp : bourgeon du pénis; ga : glande à albumen ; lp : lambeau de peau ; ov : ovotestis; spo: spermoviducte $(+$ canaux accessoires $) . \times 5$.

FIG. 2. - Coupe sagittale d'un Escargot âgé de 23 jours. Ebauche du spermoviducte (spo); progression suivie en direction du pénis (vers le bas) et en direction de la glande à albumen (vers le haut). Coloration au trichrome. (c : cœur; $p$ : poumon). $\times 40$.

FIG. 3. - Ebauche de la glande à albumen (ga) et canal hermaphrodite (ch) d'un Escargot âgé de 23 jours. Coloration au trichrome. (CBC: canal de la bourse copulatrice ; 1 : lumière du spermoviductel. $\times 280$.

FIG. 4. - U/trastructure du pénis d'un Escargot âgé d'un mois. Les noyaux (N) des cellules de l'épithélium sont allongés et riches en hétérochromatine disposée en travées. Dans le hyaloplasme, le glycogène est abondant et les mitochondries nombreuses. (lu : lumière ; me : cellules mésenchymateuses constituant la gaine du pénis) (astérisque : cellule en division). $\times 1600$.

FIG. 5. - Gonade d'un Escargot à l'éclosion. Une cellule germinale indifférenciée (cg), à gros noyau et nucléole volumineux, est bien visible. Coloration au bleu de toluidine. $\times 480$.

FIG. 6. - Gonade d'un Escargot de 11 jours. Les cellules germinales (flèches) sont disséminées entre les cellules-souches (cs) riches en polysaccharides (astérisque). Coloration au bleu de toluidine. $\times 370$.

FIG. 7. - Gonade d'un Escargot âgé d'un mois. Les cellules de la lignée mâle (sg) se trouvent dans la lumière tandis que les ovocytes (ov) sont allongés contre la paroi. (TV: tissu vésiculeux). Coloration au bleu de toluidine. $\times 370$. 

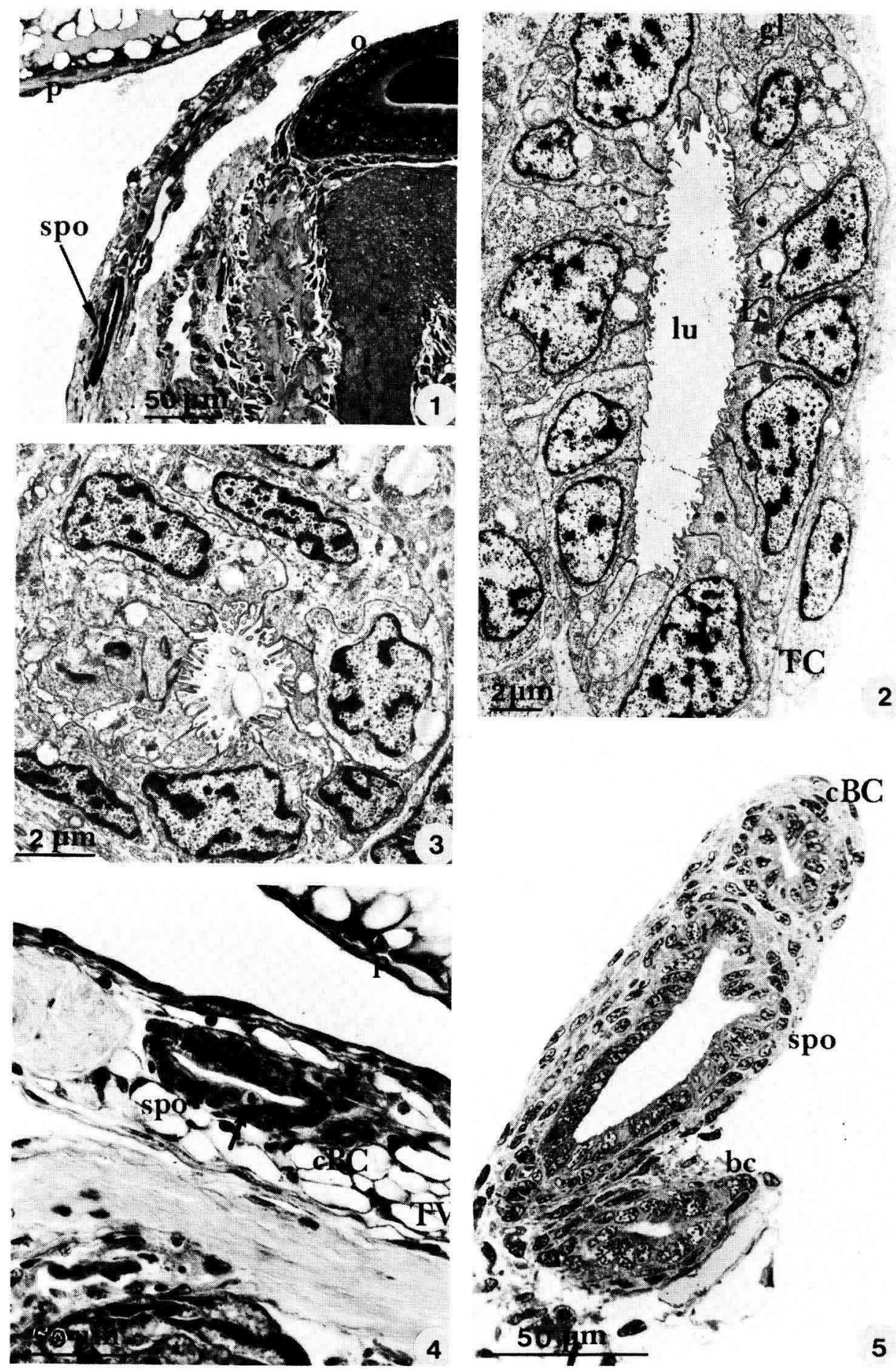
palléale. Au cours du premier mois, quelques figures de division cellulaire sont visibles au sein de l'épithélium. Vers la fin du premier mois, le spermoviducte se présente encore sous forme d'un tube aplati (fig. 2, PI. I) dont la lumière s'est légèrement élargie ; l'épithélium observé sur coupe transversale comprend 30 à 50 cellules et présente parfois quelques plis (Fig. 5, PI. II). Une couche de tissu mésenchymateux entoure le spermoviducte et le sépare des canaux accessoires à présent différenciés, tandis que le tissu vésiculeux devient abondant sur toute la longueur du tractus (fig. 4, PI. II).

b) Etude ultrastructurale. L'ébauche du spermoviducte observée à l'éclosion en microscopie électronique (fig. 2, PI. II) présente un contour régulier. Les cellules épithéliales hautes de 4 à $5 \mu \mathrm{m}$ reposent sur une lame basale très fine. Le noyau, nucléolé, assez polymorphe, occupe pratiquement tout le volume cellulaire. Les particules d'euchromatine sont dispersées, mais apparaissent plus concentrées autour de l'hétérochromatine qui constitue des masses éparses et également une couronne contre la membrane interne de l'enveloppe nucléaire. Le cytoplasme renferme du glycogène dispersé, des gouttelettes lipidiques et de rares lames d'ergastoplasme. L'appareil de Golgi est très peu développé, on relève la présence d'un ou deux petits dictyosomes. L'observation de cils en formation est peu fréquente. La membrane apicale est munie de quelques microvillosités, la zone supérieure des membranes latérales présente des jonctions du type adherens suivies de jonctions septées, et à la base des cellules quelques hémidesmosomes sont visibles.

Le spermoviducte d'Escargots âgés d'un mois est constitué de cellules épithéliales hautes de 7 à $10 \mu \mathrm{m}$ (fig. 1, PI. III) qui reposent sur une lame basale très épaisse (environ $35 \mathrm{~nm}$ ). Cette dernière est souvent plissée car elle épouse les contours de la cellule qui émet des évaginations dans le tissu mésenchymateux contenant quelques fibres de collagène et des infiltrations nerveuses visibles dans les replis du plasmalemme basal des cellules de l'épithélium (fig. 5, PI. III). A ce

\section{PLANCHE II}

FIG. 1. - Ebauche du spermoviducte (spo) dans le plancher de la cavité palléale d'un animal âgé $d^{\prime} 1$ jour. Diamètre de la coquille: $4 \mathrm{~mm}$. (o: œil ; $p$ : poumon). Coloration au bleu de toluidine. $\times 200$.

FIG. 2. - Ultrastructure de l'ébauche tubulaire du spermoviducte à l'éclosion. (gl : glycogène ; $L$ : liposome ; lu : lumière ; TC : tissu conjonctif). $\times 3400$.

FIG. 3. - Aspect ultrastructural du canal de la bourse copulatrice chez un animal âgé de 10 jours. $\times 5300$.

FIG. 4. - Spermoviducte (spo) et canal de la bourse copulatrice (cBC) d'un Escargot âgé de 23 jours, à proximité d'un nerf. Existence de mitoses au sein de l'épithélium (flèche). ( $\mathrm{p}$ : poumon ; TV : tissu vésiculeux). Coloration au trichrome. $\times 300$.

FIG. 5. - Spermoviducte (spo), canal de la bourse copulatrice (cBC) et branche copulatrice (bc) d'un animal âgé $d^{\prime} 1$ mois (diamètre : $8,7 \mathrm{~mm}$ ). Coloration au bleu de toluidine. $\times 450$. 


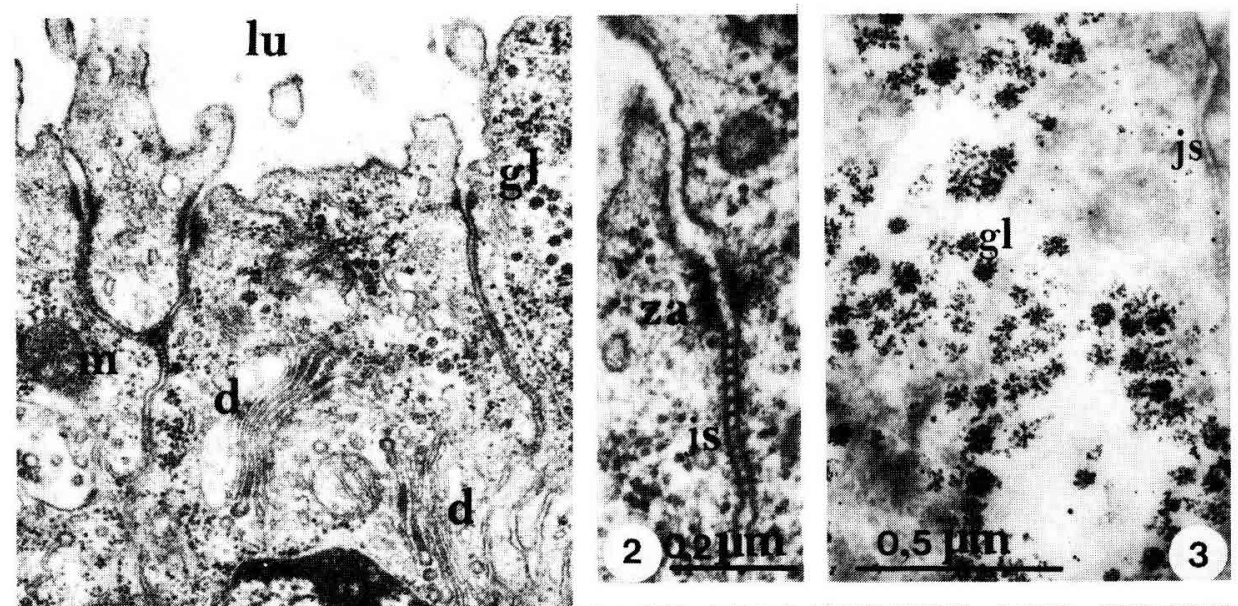

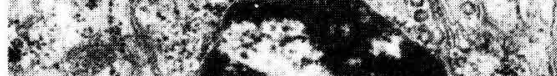

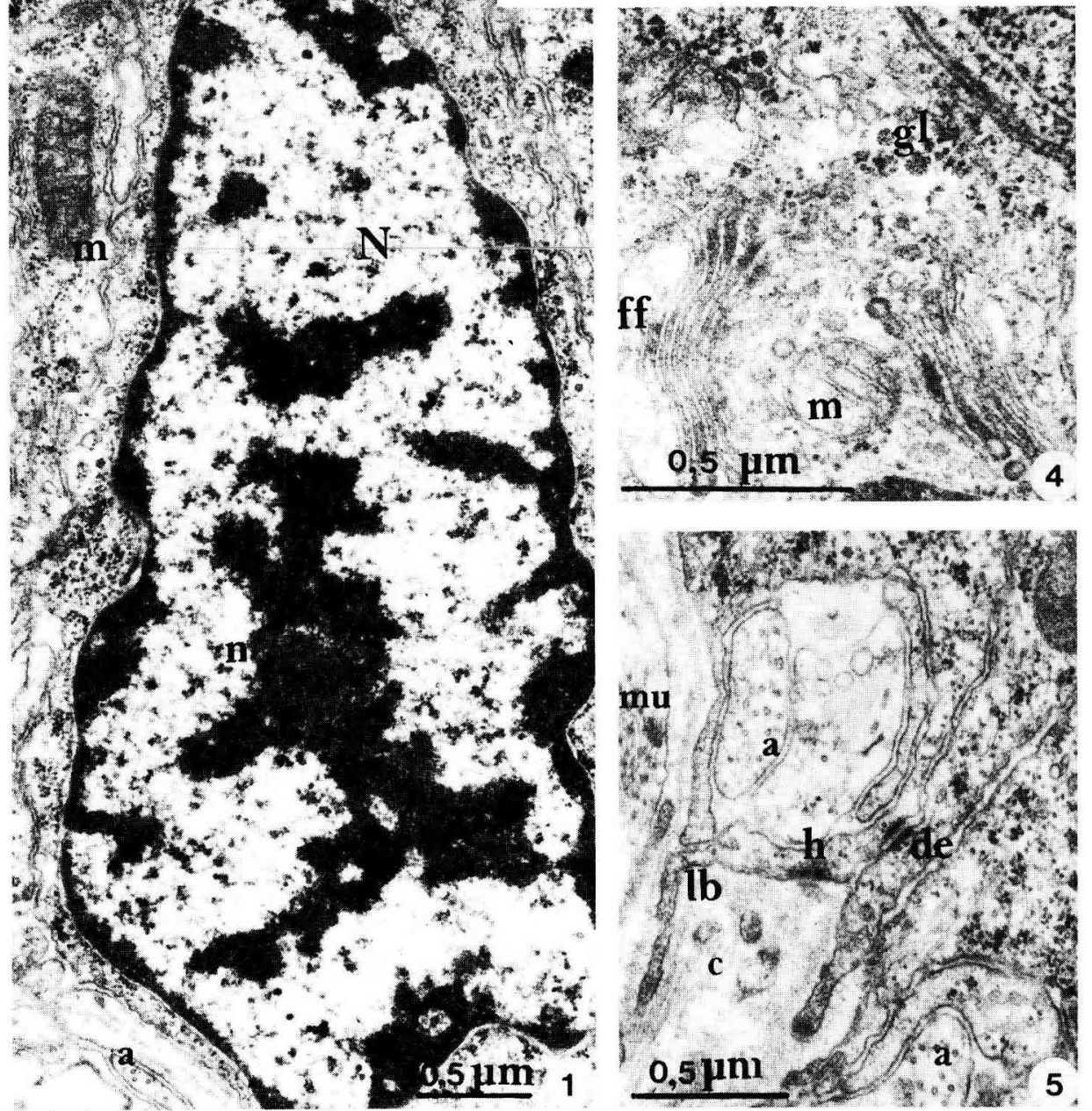


niveau, la membrane plamique présente de nombreux hémidesmosomes. De la base vers l'apex, les parois latérales des cellules épithéliales se divisent en trois zones :

- une première zone où les limites cellulaires classiques occupent pratiquement toute la paroi bien que quelques desmosomes soient présents dans la région basale (fig. 5, PI. III) ;

- une zone où apparaissent des jonctions septées, type nid d'abeilles, qui occupent la partie supérieure (au maximum $1 / 6$ de la hauteur) (fig. 2, PI III) ; - une zone située immédiatement sous la surface cellulaire apicale, où l'espace intercellulaire est élargi $(20 \mathrm{~nm})$ et les membranes épaissies. Cette ceinture autour de la cellule ou zonula adherens servirait de point d'ancrage solide et durable entre deux cellules voisines (fig. 2, PI. III).

La face apicale des cellules présente quelques courtes microvillosités. Ces évaginations digitées de la membrane sont recouvertes d'un feutrage dont la présence est inconstante : le glycocalyx. Le noyau, très allongé, occupe un volume important (fig. 1, PI. III); son grand axe est parallèle à celui de la cellule. L'hétérochromatine, répartie en grosses mottes dans le nucléoplasme, tapisse aussi plus ou moins régulièrement la membrane interne de l'enveloppe nucléaire. On distingue également de l'euchromatine sous forme de petits granules et de filaments très fins. Un ou deux nucléoles, en position centrale ou parfois périphérique, sont séparés de l'hétérochromatine associée par un fin halo clair. Au

\section{PLANCHE III}

Ultrastructure des cellules épithéliales du spermoviducte indifférencié d'un Escargot âgé d'un mois. Diamètre de la coquille: $10 \mathrm{~mm}$.

FIG. 1. - Le noyau (N) des cellules épithéliales est allongé parallèlement au grand axe cellulaire et présente ici un nucléole (n) en position centrale séparé de la chromatine associée par un fin halo clair. (a : axone ; $d$ : dictyosome; $\mathrm{gl}$ : glycogène ; lu : lumière ; $\mathrm{m}$ : mitochondrie). $\times 27000$.

FIG. 2. - Infrastructure de la membrane plasmique dans la partie latéro-apicale et apicale des ce/lules. Les microvillosités sont courtes et irrégulières. Les membranes latérales sont épaissies par des zonula adherens (za) suivies de jonctions septées (js). × 63000 .

FIG. 3. - Mise en évidence du glycogène par la réaction PATAg (temps de réaction avec la thiocarbohydrazide : $19 \mathrm{~h}$ ). Dans cette zone apicale, le cytoplasme renferme du glycogène (gl) principalement sous forme de rosettes. (js : jonction septée). $\times 42000$.

FIG. 4. - Appareil de Golgi : deux dictyosomes en position supranucléaire sont constitués de 5 à 6 saccules; ceux de la face de formation (ff) apparaissent boursouflés. Les extrémités des autres saccules renferment un matériel dense et émettent des vésicules. (gl : glycogène ; $\mathrm{m}$ : mitochondrie). $\times 55000$.

FIG. 5. - Zone basale des cellules associées à la lame basale (lb) par des hémidesmosomes (h). Les membranes plasmiques présentent des digitations en direction du mésenchyme, et sont unies latéralement par des desmosomes (de). Au sein du mésenchyme, on distingue des éléments musculaires (mu), du collagène (c) et à certains niveaux des pénétrations d'axones (a) dans les replis du plasmalemme basal des cellules épithéliales. $\times 36000$. 


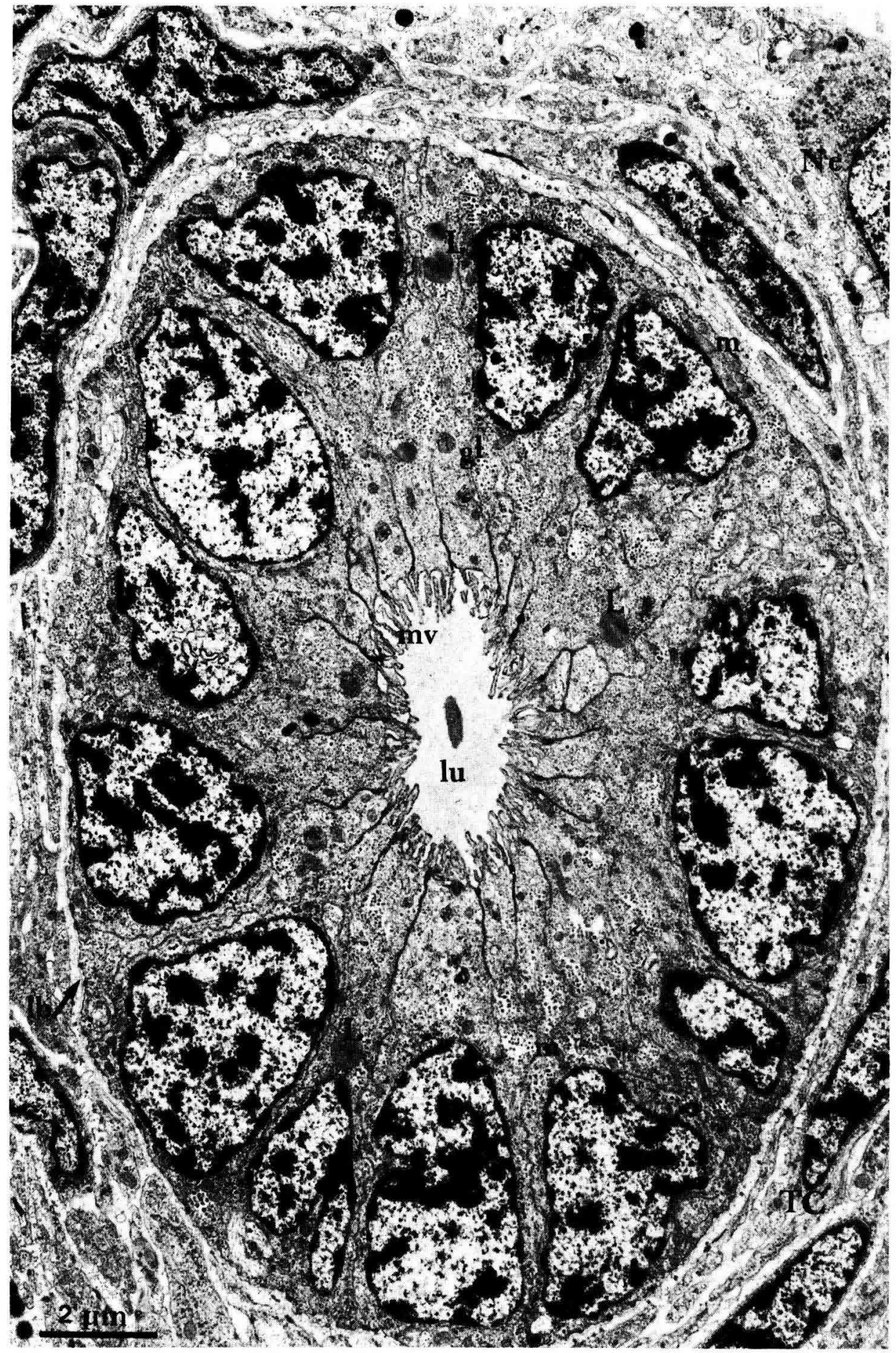


sein du hyaloplasme, le réticulum endoplasmique est peu développé ; il se localise au pôle basal et, plus fréquemment, sous forme d'une nappe le long des membranes cellulaires latérales. Des ribosomes tapissent irrégulièrement cette structure et la membrane externe du noyau. L'appareil de Golgi est formé d'un ou deux dictyosomes par cellule, en position supra-nucléaire ou légèrement latérale. Chaque dictyosome regroupe 5 à 6 saccules et la face de formation apparaît souvent dilatée (fig. 4, PI. III). Aux extrémités des saccules golgiens bourgeonnent des vésicules plus ou moins denses aux électrons. Les mitochondries, localisées principalement aux deux pôles de la cellule, renferment peu de crêtes. Le glycogène est présent dans le hyaloplasme aux pôles cellulaires, en particulier dans la région apicale. Révélé par la méthode PATAg, il se présente sous forme de rosettes caractéristiques (fig. 3, PI. III). Ce polysaccharide s'accumule en plages au niveau desquelles apparaissent quelques gouttelettes lipidiques.

5) Ebauche des canaux accessoires: canal de la bourse copulatrice et branche copulatrice. - Chez les animaux infantiles, l'apparition du premier canal

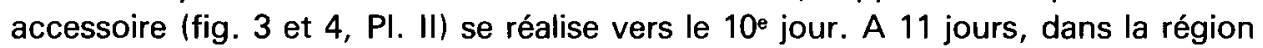
située en avant de la future glande à albumen, le tractus est constitué de deux canaux étroitement accolés, séparés par une mince assise de cellules épithéliales. Le plus large de ces canaux est le futur spermoviducte tandis que le second, de faible diamètre, correspond dans sa région inférieure à l'ébauche de la partie inférieure de la branche copulatrice et dans sa région supérieure au canal de la bourse copulatrice (renflé à son extrémité, chez les adultes, en une bourse copulatrice). A 16 jours, les ébauches du spermoviducte et du canal de la bourse copulatrice sont individualisées et séparées par une couche de tissu mésenchymateux mais, par endroits, l'épithélium des deux ébauches montre encore des zones d'étirement qui soulignent le processus de séparation des deux conduits.

En microscopie électronique, les cellules du canal de la bourse copulatrice ont le même aspect que celles de l'épithélium du spermoviducte, mis à part des microvillosités en plus grand nombre à l'apex.

Vers la fin du premier mois de vie post-embryonnaire, dans la région moyenne du tractus, on relève la présence à proximité de l'ébauche du spermoviducte d'un deuxième petit canal qui correspond à la partie supérieure de la branche copulatrice (fig. 5, PI. II). Celle-ci a généralement une section supérieure à celle du canal de la bourse. Les cellules de l'épithélium de la branche copulatrice

\section{PLANCHE IV}

Coupe transversale de la branche copulatrice d'un animal âgé d'un mois (diamètre: $10 \mathrm{~mm}$ ). Les cellules épithéliales possèdent un noyau polymorphe qui figure au sein du cytoplasme riche en glycogène ( $g l)$ et garni de quelques gouttelettes lipidiques ( $L$ ). Les mitochondries (m) sont principalement en position infra ou supra-nucléaire. La lumière (lu) est bordée de microvillosités (mv) ; la lame basale (lb) qui circonscrit l'épithélium est entourée par le tissu conjonctif (TC) dans lequel on relève la présence d'éléments nerveux (Ne). $\times 8000$. 
(PI. IV) ont les mêmes caractères ultrastructuraux que les cellules du spermoviducte mais les microvillosités sont plus abondantes et les noyaux moins allongés.

\section{Discussion.}

1) Origine de l'appareil génital. - Depuis un siècle, nombreux sont les auteurs qui ont recherché l'origine de l'appareil génital des Gastéropodes Pulmonés. Les résultats de leurs travaux ont donné naissance à une alternative entre deux théories (Martoja, 1964) : la théorie uniciste formule que l'ensemble de l'appareil reproducteur provient d'une ébauche unique, soit mésodermique (Von Jehring, 1875), soit ectodermique (Rouzaud, 1885 ; Hoffmann, 1922 ; Laviolette, 1954 ; Hochpöchler, 1979) ; la théorie dualiste soutient l'existence de deux ébauches distinctes dans l'édification de l'appareil génital. Ancel (1903), Buresch (1912), Pabst (1914), Fraser (1946), Brisson et Regondaud (1971) distinguent une ébauche mésodermique qui engendre la gonade et le canal hermaphrodite, et une ébauche ectodermique qui donne naissance au reste du tractus génital.

Les travaux entrepris sur des espèces différentes ont abouti à des controverses, et l'incertitude quant à l'origine de la gonade lectodermique, mésodermique ou combinaison de ces deux possibilités) subsiste encore aujourd'hui, comme le rappelle Runham (1982) ; notons que chez Helix aspersa, l'incorporation à la gonade de cellules mésodermiques a été observée durant les jours qui suivent la naissance (Griffond et Bride, 1981).

En revanche, la majorité des auteurs sont en accord pour attribuer au tractus une origine ectodermique, mais selon certains (Filhol, 1938 ; Runham et Laryea, 1968 ; Runham, 1978), c'est l'infiltration d'éléments mésenchymateux entre les cellules épithéliales qui est à l'origine des cellules sécrétrices des glandes annexes du tractus génital. Chez Helix aspersa, au cours du premier mois de vie postembryonnaire, nous n'avons pas observé d'infiltrations nettes de cellules mésodermiques au sein de l'épithélium des différentes parties du tractus.

2) Mise en place des ébauches génitales. - Que ce soit chez les Basommatophores qui réalisent une forme diaulique (les voies efférentes mâle et femelle se séparent sur un long parcours jusqu'aux pores génitaux distincts) ou chez les Stylommatophores qui organisent une forme monaulique (l'orifice génital est commun aux voies mâle et femelle qui s'individualisent seulement dans la région inférieure), il apparaît que la formation des différents organes génitaux se réalise par deux processus fondamentaux : le bourgeonnement et la fissuration d'une ébauche primitive tubulaire (Martoja, 1964). Les travaux de Hochpöchler (1979), Hochpöchler et Kothbauer (1979) confirment ces modalités de constitution génitale et suggèrent une origine triaule de l'appareil génital des Hélicidés en raison de la position et du développement de la branche copulatrice.

Durant le premier mois de la vie post-embryonnaire d'Helix aspersa, on assiste à la mise en place de l'ovotestis et du bourgeon pénien qui se situent respectivement aux extrémités supérieure et inférieure d'une ébauche 
ectodermique tubulaire. Une première fissuration longitudinale (10 jour) s'établit dans la région moyenne de cette ébauche tubulaire jusqu'au niveau du futur bourgeon de la glande à albumen. Alors que chez cette espèce, Rouzaud (1885) décrit la branche copulatrice comme une formation qui s'allonge au cours du développement, nos observations suggèrent que celle-ci est issue d'une deuxième séparation longitudinale comme l'indiquent Hochpöchler et Kothbauer (1979) chez un autre Hélicidé, Arianta arbustorum.

3) Aspects ultrastructuraux des cellules de l'appareil génital post-éclosion. Chez Helix aspersa, au cours du premier mois, des similitudes dans l'ultrastructure des ébauches génitales sont à noter : la cohésion cellulaire est assurée par des jonctions du type adherens suivies de jonctions septées. L'apex des cellules est hérissé de courtes microvillosités. Les cellules des différentes régions sont peu différenciées : que l'on considère les cellules-souches de l'ovotestis ou les cellules de l'épithélium du tractus, on remarque qu'elles ont un rapport nucléocytoplasmique élevé; leur pauvreté en organites et l'abondance du glycogène constituent des caractères de cellules embryonnaires. Enfin des cellules mésenchymateuses viennent partout s'appliquer contre la lame basale qui limite l'appareil reproducteur.

Contrastant avec les cellules à caractère embryonnaire, dans la gonade, des cellules germinales sont présentes dès la naissance ; pour connaître leur origine, il est à présent nécessaire de réaliser une étude approfondie du développement embryonnaire.

L'aspect sensiblement identique des cellules épithéliales dans les diverses formations tububaires de l'appareil génital (spermoviducte, canaux,...) semble en relation avec leur mise en place à partir d'une ébauche unique. Au niveau cellulaire, le tractus génital évolue peu durant le premier mois de la vie des Escargots; cependant, l'apparition de cils et l'allongement des noyaux parallèlement au grand axe des cellules dans le spermoviducte constituent les premiers signes d'une différenciation que nous nous proposons de décrire dans une publication ultérieure.

\section{Conclusion.}

Cette étude effectuée chez Helix aspersa apporte des éléments de réponse aux questions qui subsistent sur l'origine et les modalités d'organogenèse de l'appareil génital :

- Alors que des cellules mésodermiques participent à l'élaboration de la gonade, nous $\mathrm{n}^{\prime}$ avons pas constaté leur infiltration à un niveau quelconque de l'épithélium du tractus que nous considérons donc comme strictement ectodermique, au moins jusqu'à la fin du premier mois de vie post-embryonnaire.

- En ce qui concerne les processus invoqués pour rendre compte de la mise en place des ébauches génitales, il nous apparaît que l'individualisation des canaux accessoires du tractus se réalise essentiellement par fissuration d'une ébauche 
tubulaire primaire. Des bourgeonnements, locaux, se produisent ultérieurement donnant naissance, par exemple, à la glande à albumen.

Par ailleurs, nos observations soulignent le décalage existant entre l'évolution de la gonade dans laquelle tous les types cellulaires sont présents à la fin du premier mois, les gonocytes mâles et femelles étant déjà en division de méiose, et celle du tractus dont les cellules, de type épithélial, sont encore peu différenciées.

Reçu en février 1983. Accepté en mai 1983.

\section{Références}

ANCEL P., 1903. Histogenèse et structure de la glande hermaphrodite d'Helix pomatia. Arch. Biol., 19, 389-652.

ANDERSON W. A., ELLIS R. A., 1965. Ultrastructure of Trypanosoma lewisi : flagellum, microtubules and the kinetoplast. J. Protozool., 12, 483-499.

BRISSON P., REGONDAUD J., 1971. Observations relatives à l'origine dualiste de l'appareil génital chez quelques Gastéropodes Pulmonés Basommatophores. C. R. Acad. Sci. Paris, Sér. D, 273, 2339-2341.

BURESCH I., 1912. Untersuchungen über die Zwitterdrüse der Pulmonaten. I. Die Differenzierung der Keimzellen bei Helix arbustorum. Arch. Zellforsch., 7, 314-343.

COURTOT A. M., GOMOT L., 1982. Les stades de l'organogenèse et la cytodifférenciation de la glande à albumen de l'Escargot Helix aspersa Müller. Reprod. Nutr. Déve/op., 22, 661-680.

CROWELL H. H., 1973. Laboratory study of calcium requirements of the brown garden snail, Helix aspersa Müller. Proc. Malacol. Soc. London, 40, 491-503.

ENÉE J., GOMOT L., BRIDE M., 1977. Différenciation de la prostate chez l'Escargot Helix aspersa Müll. C. R. Soc. Biol., 171, 728-732.

FILHOL J., 1938. Recherches sur la nature des lépidosomes et les phénomènes cytologiquess de la sécrétion chez les Gastéropodes Pulmonés. Arch. Anat. micr., 34, 155-218 et 375-439.

FRASER L. A., 1946. The embryology of the reproductive tract of Lymnaea stagnalis appressa Say. Trans, amer. Micr. Soc., 65, 279-298.

GOMOT L., ENÉE J., 1980. Biologie de la reproduction de l'Escargot Helix aspersa Müll. : les phases de croissance et la différenciation sexuelle. Atti Acad. Fisiocritici Siena, 73-85.

GOMOT L., BRIDE J., GRIFFOND B., 1982. Participation de deux types cellulaires à la formation de l'ébauche de la gonade d'Escargot (Helix aspersa). Table ronde: Cellules germinales, XVth EDBO int. Embryol. Conf., Strasbourg.

GRIFFOND B., BRIDE J., 1981. Etude histologique et ultrastructurale de la gonade d'Helix aspersa Müller à l'éclosion. Reprod. Nutr. Dévelop., 21, 149-161.

HOCHPÖCHLER F., 1979. Vergleichende Untersuchungen über die Entwicklung des Geschlechtsapparates der Stylommatophora (Gastropoda). Zool. Anz., 202, 289-306.

HOCHPÖCHLER F., KOTHBAUER H., 1979. Triaulie bei Heliciden (Gastropoda). Zur phylogenetischen Bedeutung des Bursa copulatrix Divertikels. Z. f. zool. Syst., 17, 281-285.

HOFFMANN H., 1922. Ueber die Entwicklung der Geschlechtorgane bei Limax maximus. Zeit. Wiss. Zool., 119. 493-536.

LAVIOLETTE P., 1954. Etude cytologique et expérimentale de la régénération germinale après la castration chez Arion rufus L., Gastéropode Pulmoné. Ann. Sci. nat. Zool., 16, 427-535. 
LUFT J. H., 1961. Improvements in epoxy resin embedding methods. J. biophys. biochem. Cytol., g, $409-414$.

MARTOJA M., 1964. Développement de l'appareil reproducteur chez les Gastéropodes Pulmonés. Ann. Biol, 3, 199-232.

MARTOJA R., MARTOJA M., 1967. Initiation aux techniques de l'histologie animale. Masson, Paris.

MOLLENHAUER H. H., 1964. Plastic embedding mixtures for use in electron microscopy. Stain Technol., 39, 111-114.

PABST H., 1914. Entwicklung des Genitalapparats von Arion empiricorum. Zool. Jahrb., 38, 465-508.

REYNOLDS E. S., 1963. The use of lead citrate at high $\mathrm{pH}$ as an electron opaque stain in electron microscopy. J. Cell. Biol, 17, 208-212.

ROUZAUD H., 1885. Recherches sur le développement des organes génitaux de quelques Gastéropodes hermaphrodites. Thèse Sci., Trav. Lab. Zool. Fac. Sci. Montpellier, 1, 1-133

RUNHAM N. W., 1978. Reproduction and its control in Deroceras reticulatum. Malacologia, 17, 341-350.

RUNHAM N. W., 1982. Hermaphroditism in the Stylommatophora. Malacologia, 22, 121-123.

RUNHAM N. W., LARYEA A. A., 1968. Studies on the maturation of the reproductive system of Agriolimax reticulatus. Malacologia, 7, 93-108.

TARDY M. J., 1970. Organogenèse de l'appareil génital chez les Mollusques. Bull. Soc. Zool. Fr., 95, 407-428.

THIÉRY J. P., RAMBOURG A., 1974. Cytochimie des polysaccharides. J. Microscopie, 21, $225-232$.

TRUMP B. F., SMUCKLER E. A., BENDITT E. P., 1961. A method for staining epoxy sections for light microscopy. J. U/trastruct. Res., 5, 343-345.

VON JEHRING H., 1875. Ueber die Entwicklungsgeschichte von Helix. lena Z. Naturwiss., 9, 299-336. 